Check for updates

Cite this: RSC Adv., 2019, 9, 26850

\title{
Modifications of the CZTSe/Mo back-contact interface by plasma treatments $\uparrow$
}

\author{
Wenjian Chen, (D) *a Teoman Taskesen, (D) a David Nowak, ${ }^{a}$ Ulf Mikolajczak, ${ }^{a}$ \\ Mohamed H. Sayed, (D) a Devendra Pareek, (D) a Jörg Ohland, ${ }^{a}$ Thomas Schnabel, (D) ${ }^{b}$

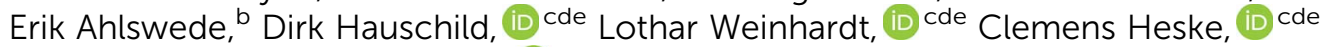 \\ Jürgen Parisia and Levent Gütay (D) ${ }^{\text {a }}$
}

\begin{abstract}
Molybdenum (Mo) is the most commonly used back-contact material for copper zinc tin selenide (CZTSe)based thin-film solar cells. For most fabrication methods, an interfacial molybdenum diselenide $\left(\mathrm{MoSe}_{2}\right)$ layer with an uncontrolled thickness is formed, ranging from a few tens of nm up to $\approx 1 \mu \mathrm{m}$. In order to improve the control of the back-contact interface in CZTSe solar cells, the formation of a MoSe $\mathrm{Mlayer}$ with a homogeneous and defined thickness is necessary. In this study, we use plasma treatments on the as-grown Mo surface prior to the CZTSe absorber formation, which consists of the deposition of stacked metallic layers and the annealing in selenium (Se) atmosphere. The plasma treatments include the application of a pure argon (Ar) plasma and a mixed argon-nitrogen $\left(\mathrm{Ar}-\mathrm{N}_{2}\right)$ plasma. We observe a clear impact of the Ar plasma treatment on the $\mathrm{MoSe}_{2}$ thickness and interfacial morphology. With the $\mathrm{Ar}-\mathrm{N}_{2}$ plasma treatment, a nitrided Mo surface can be obtained. Furthermore, we combine the Ar plasma treatment with the application of titanium nitride (TiN) as back-contact barrier and discuss the obtained results in terms of $\mathrm{MoSe}_{2}$ formation and solar cell performance, thus showing possible directions of back-contact engineering for CZTSe solar cells.
\end{abstract}

Received 15th April 2019

Accepted 19th August 2019

DOI: $10.1039 / c 9 r a 02847 a$

rsc.li/rsc-advances of $\mathrm{nm}$ up to $\approx 1 \mu \mathrm{m}$ is commonly observed in most fabrication methods. ${ }^{9-11}$

With Mo as the back-contact material, $\mathrm{MoSe}_{2}$ can form not only by a direct reaction of Mo and excess Se during absorber deposition, but also by decomposition of the CZTSe absorber at the CZTSe/Mo interface due to the difference in formation enthalpies. ${ }^{12} \mathrm{MoSe}_{2}$ is considered to have the advantages of improving adhesion and lowering the existing potential barrier at the back-contact interface., ${ }^{2,13}$ However, a too thick $\mathrm{MoSe}_{2}$ interfacial layer can cause additional series resistance and mechanical instability in the device structure. Consequently, the suppression or the control of $\mathrm{MoSe}_{2}$ formation appears to be a useful approach for improving the overall quality of the back contact and its interface with the absorber.

Methods for limiting the $\mathrm{MoSe}_{2}$ formation can be mainly divided into two groups: controlling the temperature during CZTSe formation by a multiple-step thermal process, ${ }^{\mathbf{1 4}}$ and suppression of Se diffusion by back-contact barriers. ${ }^{15-18}$ In this study, we combine the second direction with plasma treatments, striving to control the $\mathrm{MoSe}_{2}$ formation without changing our standard absorber fabrication process. This allows for reproducing our standard fabrication process for high-quality CZTSe absorbers, ${ }^{19}$ while the plasma treatments on the Mo surfaces are adopted as independent steps prior to the formation of the CZTSe absorbers. 
In detail, a plasma treatment with pure Ar is performed on the as-grown Mo surface in order to improve the homogeneity of the back-contact interface that forms during selenization. Furthermore, we perform a mixed $\mathrm{Ar}-\mathrm{N}_{2}$ plasma treatment on the as-grown Mo surface to attempt the formation of $\mathrm{Mo}_{x} \mathrm{~N}_{y}$ as a back-contact barrier to suppress $\mathrm{MoSe}_{2}$ formation. Such a nitrided Mo surface was discussed in literature to possibly have beneficial effects on interface stability and quality in thinfilm CIGS solar cells. ${ }^{2,20}$ Finally, as comparison, we combine the Ar plasma treatment with the application of TiN back-contact barrier, which was demonstrated as a possible se barrier in literature. ${ }^{15,16}$ We discuss our results in terms of $\mathrm{MoSe}_{2}$ thickness, thickness homogeneity and resulting solar cell performance.

\section{Experimental}

\section{Sample preparation}

In this study, three types of back-contact structures were prepared: standard Mo, Mo/TiN and Mo/TiN/Mo. A standard Mo layer was sputtered onto soda-lime-glass (SLG) by an $\mathrm{Ar}$ plasma with a power density of $6.1 \mathrm{~W} \mathrm{~cm}^{-2}$ at a pressure of 2.7 $\times 10^{-3} \mathrm{mbar}$, consisting of two sub-layers with a thickness of $\approx 275 \mathrm{~nm}$ for each. For Mo/TiN and Mo/TiN/Mo back contacts, a TiN layer $(\approx 10 \mathrm{~nm}$ ) was sputtered onto the standard Mo layer by an Ar plasma with a power density of $1.9 \mathrm{~W} \mathrm{~cm}^{-2}$ at a pressure of $2.7 \times 10^{-3} \mathrm{mbar}$. For the Mo top layer in the Mo/TiN/Mo case, a $50 \mathrm{~nm}$ Mo layer was sputtered onto the as-grown Mo/TiN layer under the same conditions as the standard Mo film.

For the desired modification and improvement of as-grown Mo surfaces, two types of plasma treatments were performed in this study: a pure Ar plasma treatment and a mixed $\mathrm{Ar}-\mathrm{N}_{2}$ plasma treatment. Pure Ar plasma treatments were performed with a power of $100 \mathrm{~W}$ at a pressure of $5 \times 10^{-3}$ mbar for 180 seconds. The substrates for Ar plasma treatments in this study were Mo, Mo/TiN and Mo/TiN/Mo (Fig. 1(a), (d) and (e)). They were kept at room temperature during the plasma process. The $\mathrm{Ar}-\mathrm{N}_{2}$ plasma treatments were performed by using two different (a)

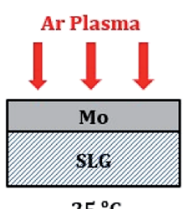

$25^{\circ} \mathrm{C}$ (b)

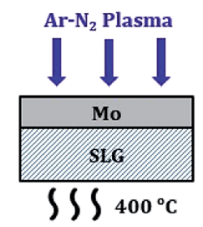

(c)

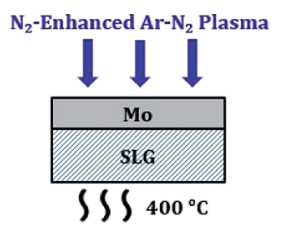

(d)

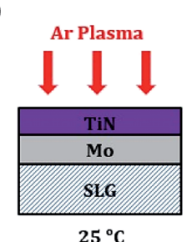

(e)

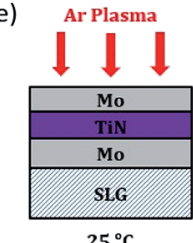

Fig. 1 Back-contact modifications with plasma treatments in this study: (a) Mo with Ar plasma, (b) Mo with $\mathrm{Ar}-\mathrm{N}_{2}$ plasma, (c) Mo with $\mathrm{N}_{2}-$ enhanced $\mathrm{Ar}-\mathrm{N}_{2}$ plasma, (d) Mo/TiN with Ar plasma and (e) Mo/TiN/ Mo with Ar plasma. sets of process parameters: (1) under standard conditions but with added $\mathrm{N}_{2}$ and longer duration of $20 \mathrm{~min}$ (atomic percentage for $\mathrm{Ar}-\mathrm{N}_{2}: 75-25 \%$ ), which we refer to as standard mixed process in the following, and (2) under enhanced $\mathrm{N}_{2}$ admixture, plasma power, and process time (atomic percentage for $\mathrm{Ar}-\mathrm{N}_{2}: 50-50 \%, 150 \mathrm{~W}, 60 \mathrm{~min}$ ), which we refer to as the $\mathrm{N}_{2}$ enhanced mixed process. The Mo substrates for both mixed $\mathrm{Ar}-$ $\mathrm{N}_{2}$ plasma treatments were heated up to $400{ }^{\circ} \mathrm{C}$ during the plasma process (Fig. 1(b) and (c)).

For all types of back contacts with and without plasma treatments, a standard fabrication process for absorber, window layer, and front contact has been established in our lab. ${ }^{11,19}$ In detail, precursors with a $\mathrm{Zn} / \mathrm{Cu}-\mathrm{Sn} / \mathrm{Zn}$ structure were deposited onto the back contacts by DC-sputtering at room temperature. The samples were then placed in a semi-closed graphite box, together with selenium (Se) pellets and tin (Sn) wire, and annealed in a conventional tube furnace at $530{ }^{\circ} \mathrm{C}$ (heating ramp: $10{ }^{\circ} \mathrm{C} \mathrm{min}^{-1}$, dwelling time: $20 \mathrm{~min}$ ). Subsequently, a cadmium sulfide (CdS) layer with a thickness of $\approx 50 \mathrm{~nm}$ was deposited onto the as-grown CZTSe absorbers $(\approx 1.2 \mu \mathrm{m})$ via a chemical bath deposition (CBD) process. Finally, $\mathrm{i}-\mathrm{ZnO}(\approx 75 \mathrm{~nm})$ and $\mathrm{Al}: \mathrm{ZnO}(\approx 550 \mathrm{~nm})$ layers were deposited by RF-sputtering as transparent front contacts. This standard fabrication process is schematically illustrated in Fig. 2. Before analysis, every sample was mechanically scribed to 9 cells with an area of $\approx 0.25 \mathrm{~cm}^{2}$ respectively. The composition of all as-grown absorbers was measured by EDX and found to be $[\mathrm{Cu}] /([\mathrm{Zn}]+[\mathrm{Sn}]) \approx 0.79 \pm 0.05$, and $[\mathrm{Zn}] /[\mathrm{Sn}] \approx 1.35 \pm 0.06$.

\section{Characterization}

To characterize the cross-section morphology of fabricated solar cells and obtain elemental composition of the as-grown absorbers, a FEI Helios Nanolab 600i Scanning Electron Microscope (SEM) combined with an Ametek ApolloX EnergyDispersive X-ray (EDX) detector was used in this study. Current-voltage $(I-V)$ characteristics were measured with a Keithley 2400 SMU under standard AM 1.5 illumination from a PET SS100AAA solar simulator. The cells were directly contacted in four-wire configuration at the $\mathrm{Al}: \mathrm{ZnO}$ and Mo layers. For understanding the impact of the mixed $\mathrm{Ar}-\mathrm{N}_{2}$ plasma treatment on the Mo surface, XPS measurements were performed in an Omicron surface science instrument using nonmonochromatized $\mathrm{Mg} \mathrm{K}_{\alpha}$ radiation from a DAR450 X-ray source and an Argus CU electron analyser. For the XPS studies, samples were transferred from Oldenburg to Karlsruhe.

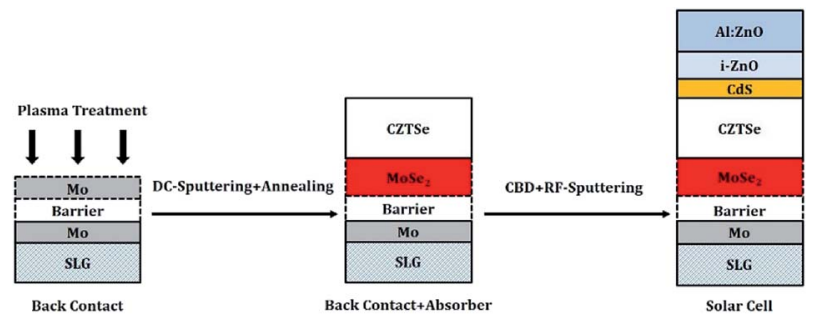

Fig. 2 Standard fabrication process of CZTSe solar cells in this study. 
To avoid surface contamination from air exposure, the samples were extracted from the processing chamber, sealed under inert nitrogen atmosphere, and introduced into the surface science instrument via glove boxes, respectively.

\section{Results and discussion}

\section{Plasma treatments on Mo back contacts}

Fig. 3(a) shows that, without plasma treatment on the Mo substrate, a $\mathrm{MoSe}_{2}$ layer is formed at the CZTSe/Mo backcontact interface, with a significant thickness variation ranging from $\approx 150 \mathrm{~nm}$ to $\approx 950 \mathrm{~nm}$. In contrast, Fig. 3(b) shows that the $\mathrm{MoSe}_{2}$ thickness is constant at $\approx 1 \mu \mathrm{m}$ for the Mo substrate, which underwent the Ar plasma treatment, while Fig. 3(c) shows that the $\mathrm{MoSe}_{2}$ thickness is constant at $\approx 900 \mathrm{~nm}$ for the Mo substrate with standard mixed $\mathrm{Ar}-\mathrm{N}_{2}$ plasma treatment. The formation of a thicker $\mathrm{MoSe}_{2}$ interfacial layer with significantly smaller local deviations for the Ar plasma-treated sample can be explained by a removal of contaminants present on the Mo surface, which could have a passivation-like behavior by blocking the Se diffusion at the Mo interface and/or directly hampering the reaction to $\mathrm{MoSe}_{2}$. For the as-grown Mo substrates, the surface could be partially contaminated with $\mathrm{O}_{2}$, $\mathrm{H}_{2} \mathrm{O}$, and organic components (e.g., residuals from the oil vapor of the vacuum pumps), which is unavoidable during storage and sample transfer through ambient conditions. They distribute randomly and possibly disturb the reaction of Se at the Mo surface during the annealing process, thus leading to a large variation in $\mathrm{MoSe}_{2}$ thickness at different positions. By the use of Ar plasma treatments, organic contaminants and weakly bonded molecules can be removed, leading to a more homogeneous and reproducible reaction during CZTSe absorber formation.

In comparison, the mixed $\mathrm{Ar}-\mathrm{N}_{2}$ plasma treatment leads to a slightly thinner $\mathrm{MoSe}_{2}$ interfacial layer than the pure $\mathrm{Ar}$

(a)
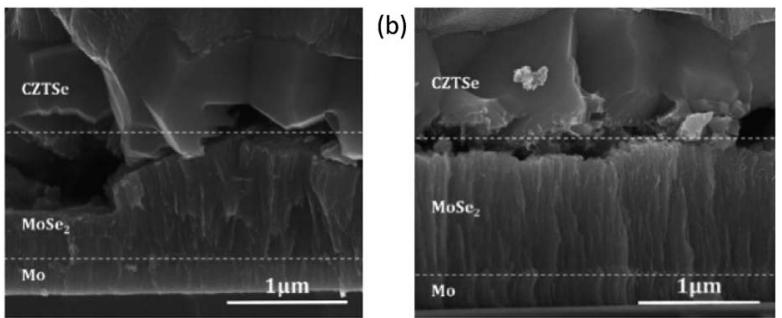

(c)

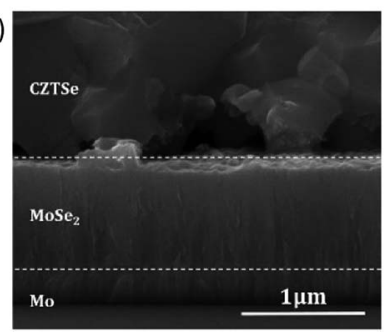

Fig. 3 SEM cross-section of CZTSe solar cells prepared on the Mo substrates (a) without plasma, (b) with Ar plasma and (c) with $\mathrm{Ar}-\mathrm{N}_{2}$ plasma treatments.

treatment does and shows a similarly small variation in thickness. The improved thickness uniformity can be ascribed to the same cleaning effect of the plasma treatment as that in the pure $\mathrm{Ar}$ case. For the sample with $\mathrm{Ar}-\mathrm{N}_{2}$ plasma treatment, a slight reduction in the $\mathrm{MoSe}_{2}$ layer thickness can likely be explained by a nitrogen-related surface passivation induced by the $\mathrm{N}_{2}$ plasma.

To confirm that the above discussed observations are related to the plasma treatment and are not significantly influenced by the presence of precursor layers, bare Mo substrates (i.e., no CZT precursor layer) were also selenized, and the SEM crosssections are shown in Fig. 4. In order to avoid excess selenization of the Mo-only samples (i.e., entire Mo layer would be selenized and no elemental Mo is left), Se amounts for these samples were reduced by half. We find that a thicker $\mathrm{MoSe}_{2}$ $(\approx 750 \mathrm{~nm}$ ) layer is formed for the sample which underwent the Ar plasma treatment compared to the sample without plasma treatment $(\approx 550 \mathrm{~nm})$. This confirms that the reduction in $\mathrm{MoSe}_{2}$ thickness observed in Fig. 3 does not have significant influence from the presence of the added CZT precursor layer, and that it is predominantly a result of the above discussed cleaning effect. Another explanation could be a microscopic roughening of the Mo surface (below the resolution limit of our observations) by material erosion due to the plasma treatment, which enhances the surface area and consequently could also lead to a higher reactivity of the surface. For the sample with Ar$\mathrm{N}_{2}$ plasma treatment, a very thin $\operatorname{MoSe}_{2}(\approx 60 \mathrm{~nm})$ layer is formed. The effect of the above-discussed possible N-related surface passivation is clearly more obvious in this case without the added precursor layer.

To study the impact of mixed Ar- $\mathrm{N}_{2}$ plasma treatments on the structure of the Mo substrates and to confirm a possible incorporation of $\mathrm{N}$ into the Mo surface, a Mo substrate without plasma treatment is compared to two $\mathrm{Ar}-\mathrm{N}_{2}$ plasma-treated samples with different $\mathrm{Ar} / \mathrm{N}$ ratios in the plasma gas, which were described in the Experimental section above as "standard

(a)
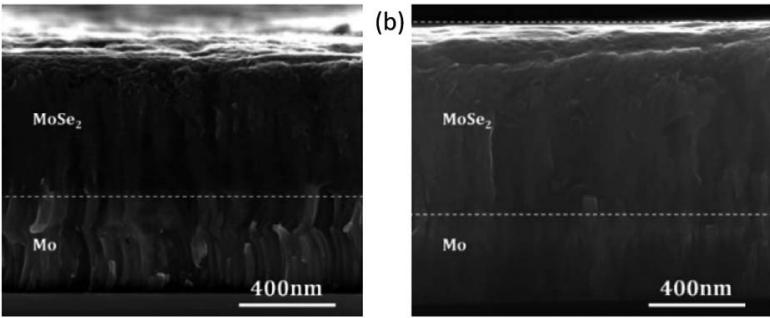

(c)

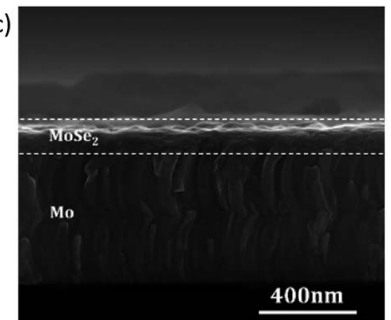

Fig. 4 SEM cross-section of selenized bare Mo substrates (a) without plasma, (b) with Ar plasma and (c) with $\mathrm{Ar}-\mathrm{N}_{2}$ plasma treatments. 
mixed" and "N-enhanced" plasma treatments. The "standard mixed" sample is identical to the sample discussed above. The $\mathrm{N}$-enhanced sample was added in order to further confirm the $\mathrm{N}$-related modification of the sample surface.

Fig. 5(a) shows the XPS survey spectra of these three samples. For all samples, Mo signals are seen (as expected), together with oxygen (O 1s at $530 \mathrm{eV}$ ) and carbon (C 1s at $285 \mathrm{eV}$ ). Small Cu, $\mathrm{Zn}$, and Sn signals can also be seen as a result of minor cross
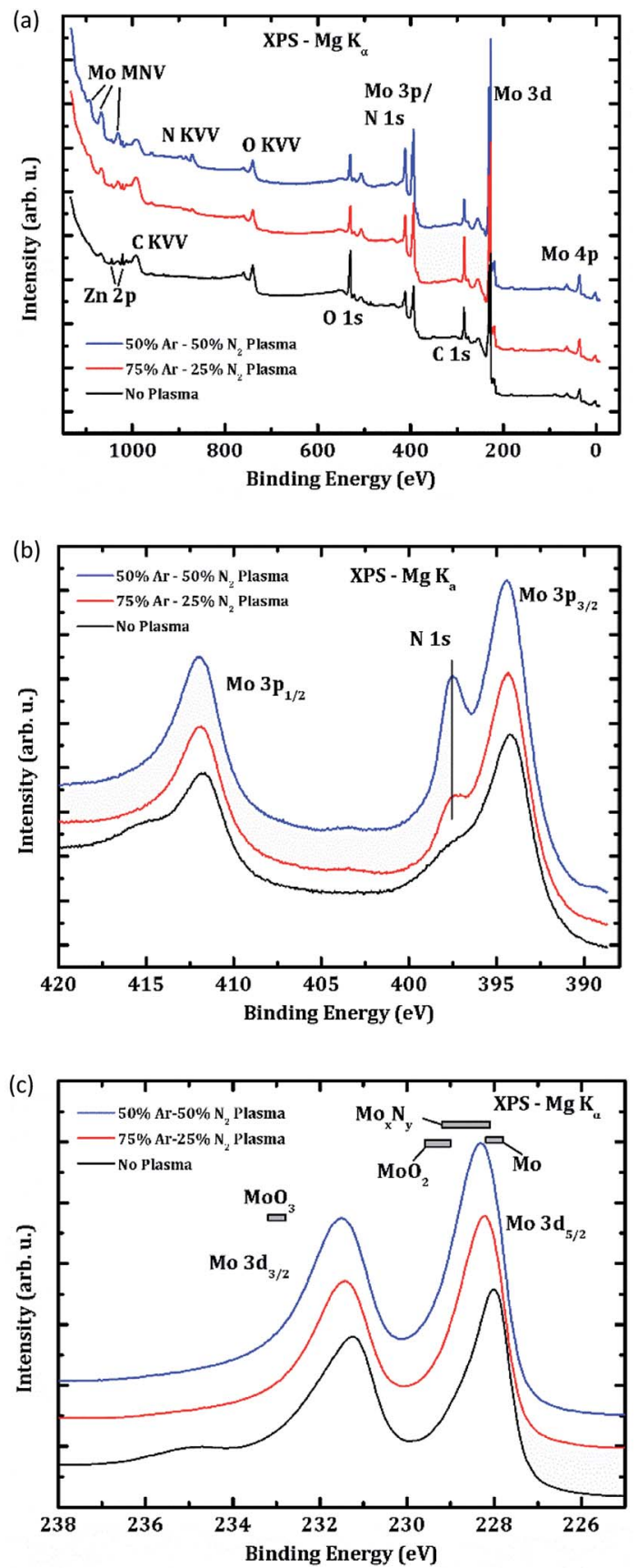

Fig. 5 (a) XPS survey spectra, (b) Mo 3p and N 1s spectrum, and (c) Mo $3 \mathrm{~d}$ spectrum of Mo substrates. Black spectra were recorded for a sample without $\mathrm{Ar}-\mathrm{N}_{2}$ plasma treatment, red and blue spectra were collected after an $\mathrm{Ar}-\mathrm{N}_{2}$ plasma treatment with an $\mathrm{Ar} / \mathrm{N}$ ratio of $75 / 25$ and 50/50, respectively. contamination in the fabrication process. The $\mathrm{Zn} 2 \mathrm{p}$ signal is most prominent and thus labeled in Fig. 5(a), but the XPS intensities of all metal contaminant signals are significantly reduced after the plasma treatments.

For both plasma-treated samples, clear N Auger-signals ( $\mathrm{N}$ $\mathrm{KVV} \sim 880 \mathrm{eV}$ ) are found, being more pronounced for the enhanced plasma treatment procedure. Furthermore, Fig. 5(b) shows a pronounced $\mathrm{N}$ 1s signal at $397.5 \mathrm{eV}$, which matches reported values for molybdenum nitrides. ${ }^{21}$ Fig. 5(b) also shows the Mo $3 p_{1 / 2}$ and $3 p_{3 / 2}$ peaks, which shift to higher binding energy after plasma treatment. Without the plasma treatment, a shoulder of the Mo peaks (at $398 \mathrm{eV}$ for Mo $3 \mathrm{p}_{3 / 2}$ and $415 \mathrm{eV}$ for Mo $3 \mathrm{p}_{1 / 2}$ ) is indicative of the presence of Mo oxide. Finally, Fig. 5(b) shows a small peak at $\sim 403 \mathrm{eV}$ for both plasma-treated samples, which we ascribe to either embedded $\mathrm{N}_{2}$ and/or $\mathrm{N}-\mathrm{O}$ bonds. ${ }^{21}$

Fig. 5(c) shows the Mo 3d signals, together with tabulated peak positions for the Mo $3 d_{5 / 2}$ peak. ${ }^{21}$ The here observed position of the "no plasma" sample fits well with metallic Mo (and also shows a characteristic metallic asymmetry), while a shoulder best seen for the Mo $3 \mathrm{~d}_{3 / 2}$ peak (at $235 \mathrm{eV}$ ) indicates the presence of Mo oxide. The Mo 3d peaks also shift towards higher binding energy for the plasma-treated samples (as expected), matching the tabulated values for $\mathrm{Mo}_{x} \mathrm{~N}_{y}$. To summarize the XPS results, we find a removal of Mo oxides, the formation of a $\mathrm{Mo}_{x} \mathrm{~N}_{y}$ phase at the surface, and a small contribution of molecular $\mathrm{N}_{2}$ or $\mathrm{N}-\mathrm{O}$ bonds after the $\mathrm{Ar}-\mathrm{N}_{2}$ plasma treatments.

To analyze the impact of the different treatments on resulting device performance, solar cells were fabricated from untreated, Ar treated, and "standard mixed" plasma treated Mo substrates. The obtained efficiencies all ended up in the range of the usual reproducibility of our process and varied between $9.5 \%$ and $11.5 \%$ device efficiency, with no obvious trends. The results are shown and discussed in the ESI, $\uparrow$ indicating a possible improvement of the sample uniformity as a result of plasma treatments and thus supporting the discussion in the previous section.

\section{Plasma treatments with TiN back-contact barrier}

The application of a TiN barrier layer on Mo back contacts was shown in literature in context of other CZTSSe fabrication methods to allow an effective reduction of $\mathrm{MoSe}_{2}$ formation. ${ }^{15,16}$ To investigate the impact of the Ar plasma treatment on the samples containing such a Se diffusion barrier layer and to obtain further insights into the impact of the overall $\mathrm{MoSe}_{2}$ thickness on the final solar cell performance, samples with a TiN back-contact barrier were also analyzed. In Fig. 6(a) and (b), SEM cross sections show a strongly reduced $\mathrm{MoSe}_{2}$ formation $\left(\mathrm{MoSe}_{2}\right.$ thickness $\left.<100 \mathrm{~nm}\right)$ for $\mathrm{Mo} / \mathrm{TiN}$ back contacts, without and with Ar plasma treatment. For another sample containing a thin Mo layer on top of the Mo/TiN back contact (i.e., Mo/TiN/Mo back-contact arrangement), only the Mo atoms located above the TiN undergo selenization and form $\mathrm{MoSe}_{2}$ (thickness constant at $\approx 230 \mathrm{~nm}$ ) (Fig. 6(c)). These results indicate that TiN works as an effective Se diffusion barrier also 
(a)
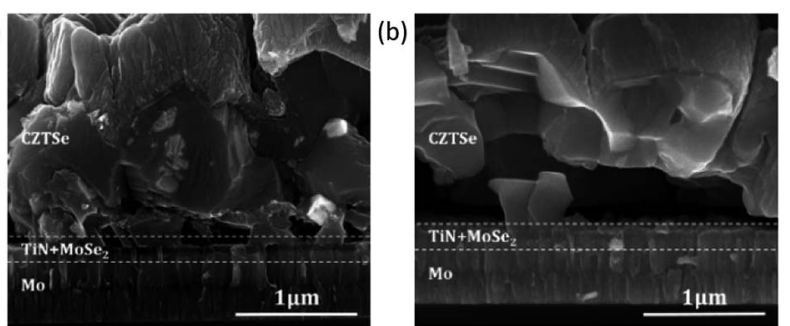

(c)

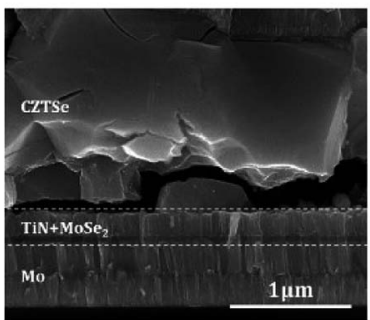

Fig. 6 SEM cross-section of CZTSe solar cells with TiN back-contact barriers: (a) Mo/TiN back contact without plasma treatment, (b) Mo/ TiN back contact with Ar plasma treatment and (c) Mo/TiN/Mo back contact with Ar plasma treatment.

in our fabrication method, and that its effect is not reduced by the applied plasma treatment.

Fig. 7 shows a comparison of the CZTSe solar cell performance with TiN back-contact barriers. In agreement with the previously reported work by Schnabel et al. ${ }^{16}$ on a solutionbased fabrication process, the Mo/TiN/Mo back-contact structure shows superior behavior compared to the two cells with $\mathrm{Mo} /$ TiN back-contact configuration. The results suggest that the direct contact of TiN with the absorber layer has a negative impact on the back-interface properties, which may result from the existence of a potential electronic barrier induced by TiN between the CZTSe absorber and the Mo back contact. For the Mo/TiN/Mo case, in which no direct contact of TiN with the CZTSe absorber exists, the negative impact of TiN is largely
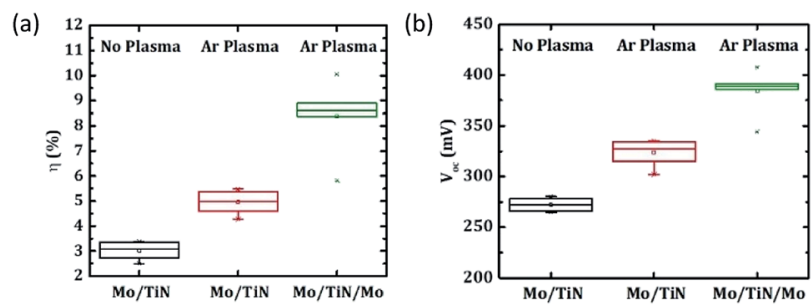

(c)

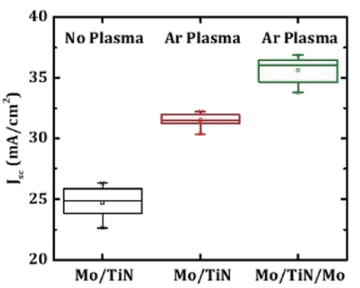

(d)

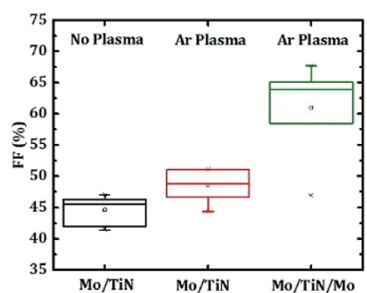

Fig. 7 Box plots of solar cell parameters (a) energy conversion efficiency $\eta$, (b) open circuit voltage $V_{o c}$, (c) short circuit current density $J_{\mathrm{sc}}$ and (d) fill factor $\mathrm{FF}$ for the samples with TiN back contact barriers. For every type of back contacts, 6 cells are included. eliminated, and the resulting CZTSe/MoSe ${ }_{2}$ interface may lead to improved electronic properties. ${ }^{2,13}$ The solar cells based on this configuration perform more closely (i.e., mainly $8-9 \%$ efficiency) to the devices without an extra back-contact barrier discussed above. However, the results do not indicate any improvement of the device properties for reducing the $\mathrm{MoSe}_{2}$ layer thickness from $\approx 1 \mu \mathrm{m}$ range down to less than $250 \mathrm{~nm}$.

\section{Conclusions}

To develop a reliable fabrication process for CZTSe-based thinfilm solar cells with efficiencies $>10 \%$, stable back-contact interfaces with a reproducible and uniform behavior are important. This becomes even more critical for upscaling in an industrial context. An Ar plasma treatment of the Mo back contact before the deposition of the CZTSe absorber can significantly improve the morphological homogeneity and thickness uniformity at the back interface. However, it also facilitates the formation of a slightly thicker $\mathrm{MoSe}_{2}$ interfacial layer between the CZTSe absorber and the Mo back contact. A modified plasma treatment with a mixed $\mathrm{Ar}-\mathrm{N}_{2}$ plasma was tested for further modification of the Mo back contact. By an appropriate adjustment of the parameters for the plasma process, a nitridation of the Mo surface (i.e., the formation of $\mathrm{Mo}_{x} \mathrm{~N}_{y}$ compounds) is possible. This procedure provides a combination of the cleaning effect under Ar plasma with the formation of a very thin $\mathrm{Mo}_{x} \mathrm{~N}_{y}$ layer that may act as a passivation layer at the back interface and lead to a reduction of $\mathrm{MoSe}_{2}$ formation during the selenization.

For CZTSe based thin film solar cells, $\mathrm{MoSe}_{2}$ appears to play a beneficial role, in terms of adhesion and band alignment between the Mo back contact and the CZTSe absorber. Unless it is too thick or too inhomogeneous in thickness to cause mechanical instability, the thickness of $\mathrm{MoSe}_{2}$ does not seem to play a crucial role for the solar cell performance. In other words, as Mo still appears to be the best choice for back contacts of CZTSe solar cells, the focus of back-contact engineering in future research should be directed away from the general thickness concern and rather towards the understanding and improvement of more decisive properties of the formed $\mathrm{MoSe}_{2}$ layer and its interface to the absorber. This may include the asgrown crystalline direction and texture, as well as the optimal energy level alignment for a heterojunction with a low hole barrier and, possibly, an electron reflector at the back contact.

\section{Conflicts of interest}

There are no conflicts to declare.

\section{Acknowledgements}

We gratefully acknowledge funding from the German Federal Ministry of Education and Research (BMBF), Free-InCa Project (grants 03SF0530A, 03SF0530B, and 03SF0530C), and the Deutsche Forschungsgemeinschaft (DFG, INST 121384/64-1 FUGG). 


\section{Notes and references}

1 T. Wada, N. Kohara, S. Nishiwaki and T. Negami, Thin Solid Films, 2001, 387, 118-122.

2 K. H. Ong, R. Agileswari, B. Maniscalco, P. Arnou, C. C. Kumar, J. W. Bowers and M. B. Marsadek, Int. J. Photoenergy, 2018, 2018, 9106269.

3 L. Weinhardt, O. Fuchs, A. Peter and E. Umbach, J. Chem. Phys., 2016, 124, 074705.

4 L. Weinhardt, M. Blum, M. Bär, C. Heske, O. Fuchs, E. Umbach, J. D. Denlinger, K. Ramanathan and R. Noufi, Thin Solid Films, 2007, 515, 6119-6122.

5 M. Bär, S. Nishiwaki, L. Weinhardt, S. Pookpanratana, W. N. Shafarman and C. Heske, Appl. Phys. Lett., 2008, 93, 042110.

6 M. Bär, L. Weinhardt and C. Heske, Phys. Rev. B: Condens. Matter Mater. Phys., 2008, 78, 075404.

7 S. Oueslati, G. Brammertz, M. Buffière, H. ElAnzeery, D. Mangin, O. ElDalf, O. Touayar, C. Köble, M. Meuris and J. Poortmans, J. Phys. D: Appl. Phys., 2015, 48, 035103.

8 P. D. Antunez, D. M. Bishop, Y. S. Lee, T. Gokmen, O. Gunawan, T. S. Gershon, T. K. Todorov, S. Singh and R. Haight, Adv. Energy Mater., 2017, 7, 1602585.

9 G. Brammertz, M. Buffière, S. Oueslati, H. ElAnzeery, K. Ben Messaoud, S. Sahayaraj, C. Köble, M. Meuris and J. Poortmans, Appl. Phys. Lett., 2013, 103, 163904.

10 Y. S. Lee, T. Gershon, O. Gunawan, T. K. Todorov, T. Gokmen, Y. Virgus and S. Guha, Adv. Energy Mater., 2014, 2014, 1401372.
11 T. Taskesen, J. Neerken, J. Schoneberg, D. Pareek, V. Steininger, J. Parisi and L. Gütay, Adv. Energy Mater., 2018, 8, 1703295.

12 A. Shigemi and T. Wada, Jpn. J. Appl. Phys., 2018, 57, 08 RC17.

13 D. Cozza, C. M. Ruiz, D. Duché, J. J. Simon and L. Escoubas, IEEE J. Photovolt., 2016, 6, 5.

14 J. Li, Y. Zhang, W. Zhao, D. Nam, H. Cheong, L. Wu, Z. Zhou and Y. Sun, Adv. Energy Mater., 2015, 5, 1402178.

15 B. Shin, Y. Zhu, N. A. Bojarczuk, S. Jay Chey and S. Guha, Appl. Phys. Lett., 2012, 101, 053903.

16 T. Schnabel and E. Ahlswede, Sol. Energy Mater. Sol. Cells, 2017, 159, 290-295.

17 S. López-Marino, M. Placidi, A. Pérez-Tomás, J. Llobet, V. Izquierdo-Roca, X. Fontané, A. Fairbrother, M. Espíndola-Rodríguez, D. Sylla, A. Pérez-Rodríguez and E. Saucedo, J. Mater. Chem. A, 2013, 1, 8338-8343.

18 M. H. Sayed, J. Schoneberg, J. Parisi and L. Gütay, RSC Adv., 2017, 7, 20406-20411.

19 T. Taskesen, V. Steininger, W. Chen, J. Ohland, U. Mikolajczak, D. Pareek, J. Parisi and L. Gütay, Prog. Photovolt. Res. Appl., 2018, 26, 1003-1006.

20 S. Uličná, P. Arnou, A. Abbas, M. Togay, L. M. Welch, M. Bliss, A. V. Malkov, J. M. Walls and J. W. Bowers, J. Mater. Chem. A, 2019, 7, 7042-7052.

21 NIST X-ray Photoelectron Spectroscopy Database, NIST Standard Reference Database Number 20, National Institute of Standards and Technology, Gaithersburg MD, 2000, p. 20899, https://srdata.nist.gov/xps/citation.aspx. 